\title{
Numerical Simulations of Viscoelastic Fluid Flows Using a Least-Squares Finite Element Method Based on Von Mises Stress Criteria
}

\author{
Hsueh-Chen Lee* \\ General Education Center, Wenzao Ursuline University of Languages, Kaohsiung, Taiwan. \\ * Corresponding author. Email: 87013@mail.wzu.edu.tw; hsuehchen.lee@gmail.com \\ Manuscript submitted March 2, 2017; accepted May 13, 2017. \\ doi: 10.17706/ijapm.2017.7.3.157-164
}

\begin{abstract}
The work concerns least-squares (LS) finite element solutions of the Oldroyd-B viscoelastic fluid flows using adaptive grids. To capture the viscoelastic flow region, adaptive grids are automatically generated on the basis of the von Mises stress from stress component transfer functions, and these generated refined grids agree with the physical flow attributes. Model problems considered are the flow past a slot channel problems. Numerical solutions of the flow pass through a slot channel indicate that the viscoelastic polymer solution flow characteristics are described by the refinement results of the von Mises stress. In additions, adaptive grids using the von Mises stress outperform those using functions of velocity, and satisfactory results are obtained using a lower total numbers of elements. The developed method is effective for analyzing viscoelastic fluid flows. Furthermore, the effects of low Weissenberg numbers are also investigated.
\end{abstract}

Key words: Least-squares, viscoelastic fluid, adaptive mesh refinement, von Mises stress, transverse Slot.

\section{Introduction}

The objective of this study is to analyze an adaptive LS approach for the Navier-Stokes equations that govern the flow of viscoelastic fluids by using the Oldroyd-B model [1]; in contrast to the Newtonian model, the problems are associated with fluid viscosity, elasticity, and memory. To simulate viscoelastic flow problems, it is convenient to split the extra-stress tensor into viscous and elastic parts by changing of variables in the LS formulations [1], [2]. The LS functional has been reported to offer several theoretical and computational advantages [3], and also provides a local and inexpensive a posteriori error estimator that may be used to guide adaptive refinement [4] and is perfectly effective and reliable for error control [5]. Therefore, difficulties in solving non-Newtonian flow problems include computational limitations and singularities caused by the high number of unknowns and geometric discontinuities, respectively. To resolve these problems, adaptive LS methods have been extensively used as powerful tools for obtaining more efficient and accurate results [6]-[8]. In [7], an adaptive refined LS approach (ALS) generated using a velocity magnitude is developed to refine the mesh adaptively for the Carreau generalized Newtonian fluid flows. As affected by stress in the viscoelastic fluid problem, the current motion of the fluid is associated with viscosity and elasticity. Therefore, the method of analysis should include some consideration of stress behavior for viscoelastic fluid flows.

On the basis of these studies, we develop an LS method based on the adaptive mesh using a stress 
magnitude to refine the mesh adaptively for the Oldroyd-B model. Note that in structural applications, the von Mises stress is often used as the metric for evaluating design margins [9]. Based on the idea, it is meaningful to use the von Mises stress as a characteristic stress magnitude as a superposition of the polymeric and viscous stresses in viscoelastic fluid flows. To capture the flow region and understand the flow feature, we modify the adaptive mesh algorithm in [7] by using the von Mises stress to refine grid points. To prevent the loss of mass conservation and convergence of numerical schemes when low-order basis functions are used, a LS method involving appropriate mass conservation weights is used in the study. Furthermore, we estimate the coercivity and continuity for the homogeneous LS functional, which involves the sum of the equation residuals measured in the $L^{2}$-norm. The analysis of the linearized viscoelastic fluid using the LS method follows the concept introduced in [1], [6]. We extend the implementation to simulate the flow through a transverse slot problem using conforming piecewise polynomial elements for all unknowns. We show that adaptive grids using the von Mises stress and the streamlines used in the approach are similar to those in [10]. We also demonstrate that the adaptive LS method agrees with the physical attributes of these models with various parameters, and satisfactory results are obtained using a lower total numbers of elements.

The rest of this paper is organized as follows. Section 2 presents the notation, preliminaries, and coercivity and continuity of the LS functional, and the uniqueness theorem for the LS approximations. Section 3 presents numerical results for the flow past a slot in a channel, and Section 4 offers concluding remarks.

\section{A least-squares finite element method}

The Oldroyd-B model is a popular viscoelastic model in polymeric fluid simulations in [1], and the extra-stress is written as a superposition of the polymeric and viscous stresses, $\boldsymbol{\tau}=\boldsymbol{\tau}_{\mathrm{p}}+\boldsymbol{\tau}_{\mathrm{s}}$. The viscous stress, which in some applications is associated with a solvent, is Newtonian, i.e., $\boldsymbol{\tau}_{\mathrm{s}}=2 \eta_{s} \mathbf{D}(\mathbf{u})$, where $\eta_{s}$ is a constant viscosity and $\mathbf{D}(\mathbf{u})=0.5\left(\nabla \mathbf{u}+\nabla \mathbf{u}^{\mathrm{T}}\right)$ is the standard strain rate tensor with the velocity $\mathbf{u}$. In the linearized form of the Oldroyd-B model, the polymer contribution to the stress obeys the following equation [1],

$$
\boldsymbol{\tau}_{\mathbf{p}}+\lambda\left(\mathbf{u} \cdot \nabla \boldsymbol{\tau}_{p}-A\left(\nabla \mathbf{u}, \boldsymbol{\tau}_{p}\right)\right)=2 \eta_{p} \mathbf{D}(\mathbf{u})
$$

where

$$
A\left(\nabla \mathbf{u}, \boldsymbol{\tau}_{p}\right)=(\nabla \mathbf{u})^{\mathrm{T}} \cdot \boldsymbol{\tau}_{p}+\boldsymbol{\tau}_{p} \cdot \nabla \mathbf{u}
$$

In (1), $\lambda$ is a relaxation time and $\eta_{p}$ is the polymeric contribution to the viscosity. Consider the steady-state, incompressible Navier-Stokes problem in a two-dimensional, $\Omega$, with boundary, collecting (1), we have the Oldroyd-B model written as

$$
\begin{aligned}
\operatorname{Re}(\mathbf{u} \cdot \nabla \mathbf{u})-\nabla \cdot \boldsymbol{\tau}_{p}-\nabla \cdot \boldsymbol{\tau}_{s}+\nabla p=\mathbf{0} & \text { in } \Omega, \\
\nabla \cdot \mathbf{u}=\mathbf{0} & \text { in } \Omega, \\
\boldsymbol{\tau}_{s}-2 \beta \mathbf{D}(\mathbf{u})=\mathbf{0} & \text { in } \Omega, \\
\boldsymbol{\tau}_{\mathbf{p}}+W e\left(\mathbf{u} \cdot \nabla \boldsymbol{\tau}_{p}-A\left(\nabla \mathbf{u}, \boldsymbol{\tau}_{p}\right)\right)-2(1-\beta) \mathbf{D}(\mathbf{u})=\mathbf{0} & \text { in } \Omega, \\
\mathbf{u}=\mathbf{0} & \text { in } \Gamma,
\end{aligned}
$$


where $R e>0$ is the Reynolds number, $R e \equiv L_{c} U_{c} \rho / \eta_{0}$, in which $\eta_{0}=\eta_{\mathrm{p}}+\eta_{\mathrm{s}}$ is viscosity, $L_{c}$ and $U_{c}$ are characteristic length and velocity, respectively. $\beta=\eta_{\mathrm{s}} / \eta_{0} \in[0,1]$ is the ratio of solvent viscosity to the total zero-shear-rate viscosity and $W e \geq 0$ is the Weissenberg number, $W e \equiv \lambda U_{c} / L_{c}$. The upper-convected Maxwell (UCM) model has $\beta=0$. In the case of $W e=0$, the model reduces to the Newtonian model, the Navier-Stokes equations.

Let the product space $\boldsymbol{\Phi}:=\mathbf{H}^{1}(\Omega)^{2} \times L^{2}(\Omega) \times \mathbf{L}^{2}(\Omega)^{2}$. Based on [1], linearizing (2)-(6) about the approximation

$$
\mathbf{u} \cdot \nabla \mathbf{u} \approx \mathbf{u}_{1} \cdot \nabla \mathbf{u}+\mathbf{u} \cdot \nabla \mathbf{u}_{1}-\mathbf{u}_{1} \cdot \nabla \mathbf{u}_{1}, \quad \boldsymbol{\tau}_{p 1} \approx \boldsymbol{\tau}_{p}, \quad \mathbf{u}_{1} \approx \mathbf{u}
$$

where we assume $\nabla \cdot \mathbf{u}_{1}=0$ and

$$
\max \left\|\mathbf{u}_{1}\right\|_{\infty}, \nabla \mathbf{u}_{1},\left\|\boldsymbol{\tau}_{1}\right\|_{\infty},\left\|\nabla \boldsymbol{\tau}_{1}\right\|_{\infty} \leq M<\infty
$$

results in the following replacement rules:

$$
\begin{gathered}
\mathbf{u} \cdot \nabla \boldsymbol{\tau}_{p} \approx \mathbf{u}_{1} \cdot \nabla \boldsymbol{\tau}_{p}+\mathbf{u} \cdot \nabla \boldsymbol{\tau}_{p 1}-\mathbf{u}_{1} \cdot \nabla \boldsymbol{\tau}_{p 1} \\
\mathrm{~A}\left(\nabla \mathbf{u}, \boldsymbol{\tau}_{p}\right) \approx \mathrm{A}\left(\nabla \mathbf{u}_{1}, \boldsymbol{\tau}_{p}\right)+\mathrm{A}\left(\nabla \mathbf{u}, \boldsymbol{\tau}_{p 1}\right)-\mathrm{A}\left(\nabla \mathbf{u}_{1}, \boldsymbol{\tau}_{p 1}\right)
\end{gathered}
$$

The linearized system of the Oldroyd-B model may now be written as

$$
\begin{aligned}
\operatorname{Re}\left(\mathbf{u}_{1} \cdot \nabla \mathbf{u}+\mathbf{u} \cdot \nabla \mathbf{u}_{1}\right)-\nabla \cdot \boldsymbol{\tau}_{p}-\nabla \cdot \boldsymbol{\tau}_{s}+\nabla p=\mathbf{f}_{1} & \text { in } \Omega, \\
\nabla \cdot \mathbf{u}=\mathbf{0} & \text { in } \Omega, \\
\boldsymbol{\tau}_{s}-2 \beta \mathbf{D}(\mathbf{u})=\mathbf{0} & \text { in } \Omega, \\
\boldsymbol{\tau}_{\mathbf{p}}+W e\left(\mathbf{u} \cdot \nabla \boldsymbol{\tau}_{p}\right)+\mathrm{B}\left(\mathbf{u}, \boldsymbol{\tau}_{p}\right)-2(1-\beta) \mathbf{D}(\mathbf{u})=\mathbf{f}_{2} & \text { in } \Omega, \\
\mathbf{u}=\mathbf{0} & \text { in } \Gamma,
\end{aligned}
$$

where we define

$$
\begin{gathered}
\mathbf{f}_{1}=\operatorname{Re}\left(\mathbf{u}_{1} \cdot \nabla \mathbf{u}_{1}\right), \\
\mathrm{B}\left(\mathbf{u}, \boldsymbol{\tau}_{p}\right)=W e\left(\mathbf{u}_{1} \cdot \nabla \boldsymbol{\tau}_{p}+\mathrm{A}\left(\nabla \mathbf{u}_{1}, \boldsymbol{\tau}_{p}\right)+\mathrm{A}\left(\nabla \mathbf{u}, \boldsymbol{\tau}_{p_{1}}\right)\right) \\
\mathbf{f}_{2}=W e\left(\mathbf{u}_{1} \cdot \nabla \boldsymbol{\tau}_{p 1}+\mathrm{A}\left(\nabla \mathbf{u}_{1}, \boldsymbol{\tau}_{p 1}\right)\right)
\end{gathered}
$$

The least-squares (LS) functional for (8)-(12) is given by

$$
\begin{aligned}
\mathbf{J}(\mathbf{U} ; \mathbf{F}) & =\left\|\operatorname{R} e\left(\mathbf{u}_{1} \cdot \nabla \mathbf{u}+\mathbf{u} \cdot \nabla \mathbf{u}_{1}\right)-\nabla \cdot \boldsymbol{\tau}_{p}-\nabla \cdot \boldsymbol{\tau}_{s}+\nabla p-\mathbf{f}_{1}\right\|_{0}^{2}+K\|\nabla \cdot \mathbf{u}\|_{0}^{2} \\
& +\left\|\boldsymbol{\tau}_{s}-2 \beta \mathbf{D}(\mathbf{u})\right\|_{0}^{2}+W\left\|\boldsymbol{\tau}_{\mathbf{p}}+W e\left(\mathbf{u} \cdot \nabla \boldsymbol{\tau}_{p}\right)+\mathrm{B}\left(\mathbf{u}, \boldsymbol{\tau}_{p}\right)-2(1-\beta) \mathbf{D}(\mathbf{u})-\mathbf{f}_{2}\right\|_{0}^{2},
\end{aligned}
$$

$\forall \mathbf{U}=\left(\mathbf{u}, p, \boldsymbol{\tau}_{s}, \boldsymbol{\tau}_{p}\right) \in \boldsymbol{\Phi}$. The weights of positive constants $K$ and $W$ are chosen on the basis of similar 
considerations as those in [11]. In [11], the results indicate that LS solutions can be improved by sufficiently scaling the conservation and constitutive equations.

Denote the norm as

$$
\|\mathbf{U}\|=\left(\|\mathbf{u}\|_{1}^{2}+\|p\|_{0}^{2}+\left\|\boldsymbol{\tau}_{s}\right\|_{0}^{2}+\left\|\boldsymbol{\tau}_{p}\right\|_{0}^{2}+\left\|\mathbf{u}_{1} \cdot \nabla \boldsymbol{\tau}_{p}\right\|_{0}^{2}\right)^{1 / 2}
$$

$\forall \mathbf{U} \in \mathbf{\Phi}$. Based on [1], we can establish an a priori estimate for the functional in Theorem 1 as shown in [12].

Theorem 1. For any $\mathbf{U} \in \mathbf{\Phi}$, there is a positive constant $C$ which depends on $\Omega, \beta, W e$, Re, and $M$ in (7), such that

$$
C\|\mathbf{U}\|^{2} \leq \mathbf{J}(\mathbf{U} ; \mathbf{0}) \leq C\|\mathbf{U}\|^{2}
$$

for sufficiently small values of $M, W e$, and $R e$.

Therefore, the coercivity and continuity estimates of the functional $\mathrm{J}$ have been established in Theorem 1. The LS minimization problem for the solution of system (8)-(12) is to choose $\mathbf{U} \in \mathbf{\Phi}$ such that

$$
\mathbf{J}(\mathbf{U} ; \mathbf{F})=\inf _{\mathbf{V} \in \Phi} \mathbf{J}(\mathbf{V} ; \mathbf{F})
$$

For the finite element approximation, we assume that the domain $\Omega$ is a polygon and that $T_{h}$ is a partition of $\Omega$ into finite elements $\Omega=\bigcup_{T \in T_{h}} T$ with $h=\max \left\{\operatorname{diam}(T): T \in T_{h}\right\}$. Let $\boldsymbol{\Phi}^{h}$ be finite element subspaces of $\boldsymbol{\Phi}$. The discrete minimization problem is to choose $\mathbf{U}^{h} \in \boldsymbol{\Phi}^{h}$ such that

$$
\mathbf{J}(\mathbf{U} ; \mathbf{F})=\inf _{\mathbf{v}^{h} \in \Phi^{h}} \mathbf{J}\left(\mathbf{V}^{h} ; \mathbf{F}\right)
$$

Since $\boldsymbol{\Phi}^{h} \subset \boldsymbol{\Phi}$, using arguments similar to those in [1], and Theorem 1, and the Lax-Milgram lemma, the uniqueness theorem is proved.

Theorem 2. If the problem (8)-(12) has a unique solution $\mathbf{U} \in \boldsymbol{\Phi}$. Then there exists a unique solution $\mathbf{U}^{h} \in \boldsymbol{\Phi}^{h} \subset \boldsymbol{\Phi}$ satisfying (16) for sufficiently small values of $M$ in (17).

\section{Numerical results}

The governing equations are solved on one domain in this section. The domain is the flow of the Oldroyd-B model past a slot in a channel. Our method couples the LS method (13) with the adaptively refined algorithms presented in [7] for the generalized Newtonian fluid flows. In the work, to capture the flow region of the Oldroyd-B model, we employ the adaptive mesh algorithm in [7], using the grading function of the least-squares solutions such as velocity magnitude $|\mathbf{u}|_{2}=\sqrt{\mathbf{u}_{x}^{2}+\mathbf{u}_{y}^{2}}$ and von Mises stress,

$$
\boldsymbol{\tau}_{v}=\sqrt{0.5\left(\boldsymbol{\tau}_{x x}^{2}+\boldsymbol{\tau}_{y y}^{2}-\boldsymbol{\tau}_{x x} \boldsymbol{\tau}_{y y}+3 \boldsymbol{\tau}_{x y}^{2}\right)}
$$

as shown in [9]. The LS method with the adaptive algorithm using the grading function of $|\mathbf{u}|_{2}$ and $\boldsymbol{\tau}_{v}$ are

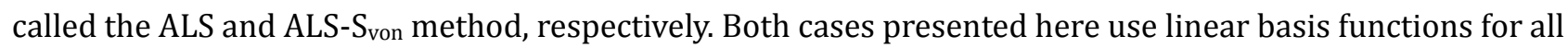


variables.

To further show the capability of the numerical algorithm in the Oldroyd-B model, we apply the method to the flow in a slot channel shown in Figure 1; $x$ (the flow direction) varied from $-2.5 \leq x \leq 2.5$ and the central line of the slot occurred at $x=0$. The boundary conditions include those at the inlet, outlet and wall boundaries. The velocity $\mathbf{u}_{y}=0$ is specified on the inflow and pressure $p$ is set to zero at the point where the outflow boundary meets the wall. These boundary conditions are also used in [10]. In (8)-(12), the parameters $(R e, \beta, 1-\beta, W e)$ are required to describe the fluid rheology. In our results, the parameters are set as $\operatorname{Re}=1, \quad \beta=1 / 9$, and $W e=0.2$.
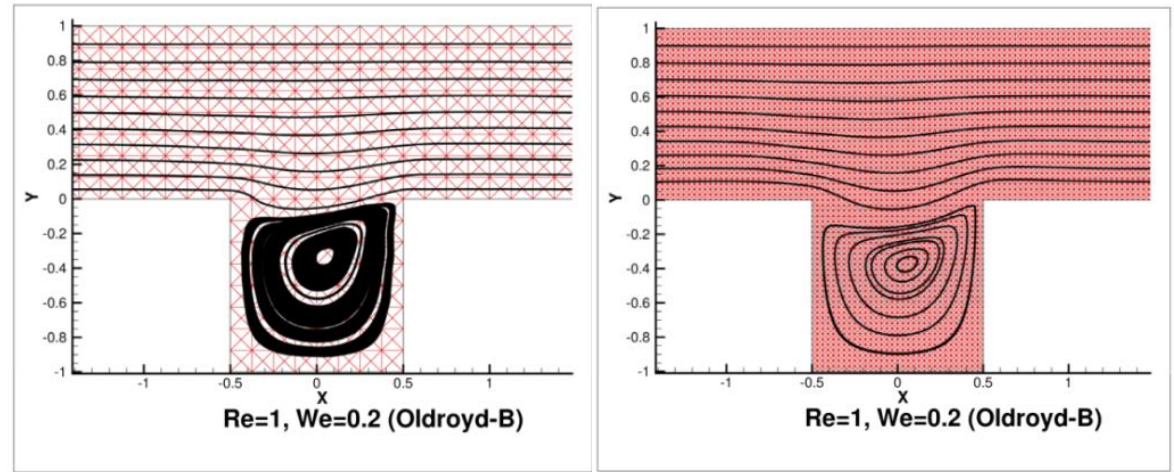

(a) Mesh 1 with 1056 elements using the LS (b) Mesh 2 with 24576 elements using the LS
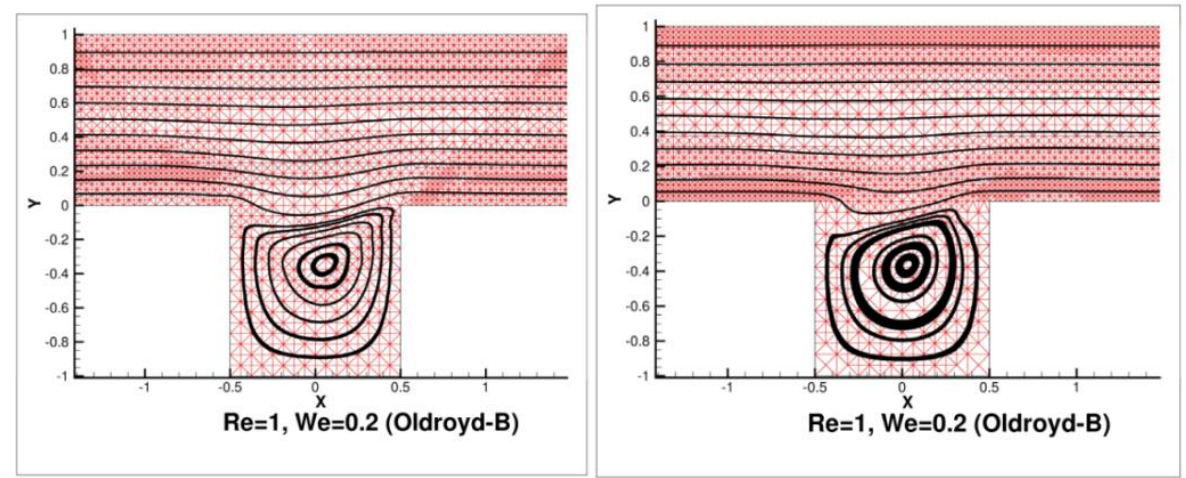

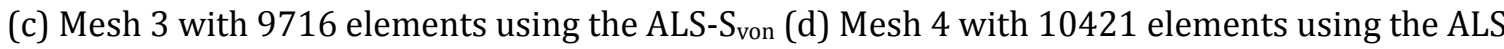

Fig. 1. Streamlines of the LS method using uniform Meshes (a) 1 and (b) 2, and adaptive Meshes (c) 3 and (d) 4 for $R e=1$ and $W e=0.2$.

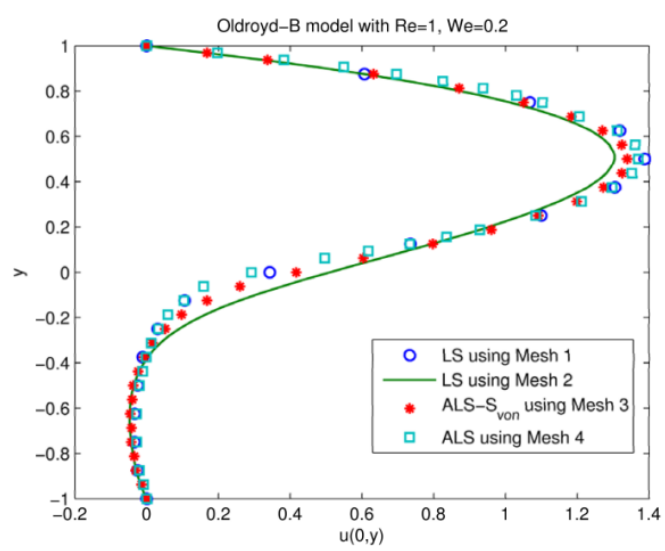

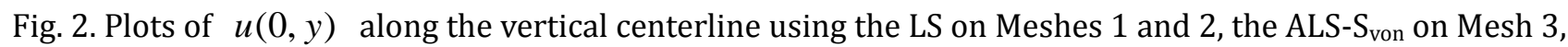
and the ALS on Mesh 4 for $R e=1$ and $W e=0.2$. 


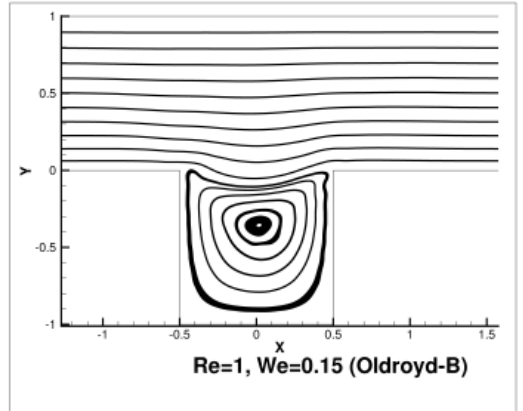

(a)

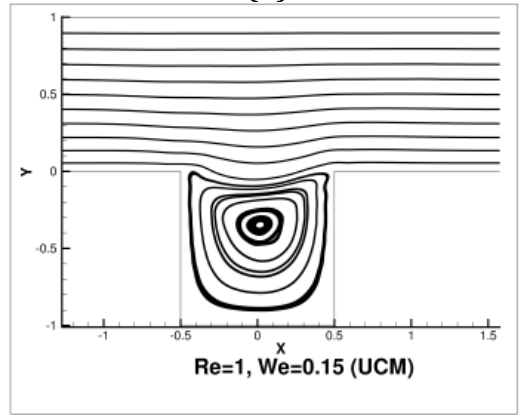

(d)

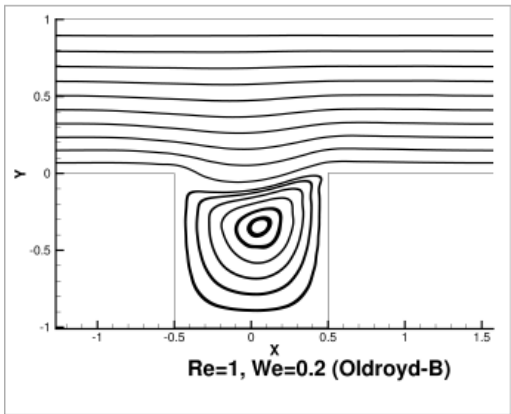

(b)

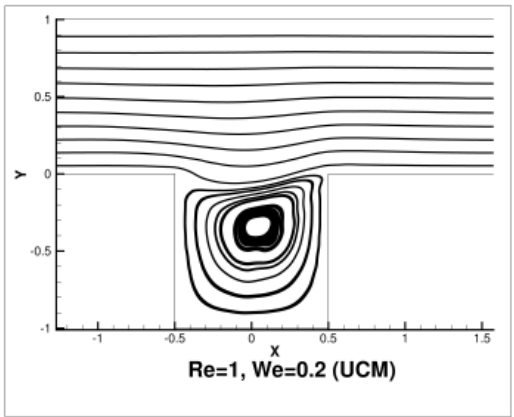

(e)

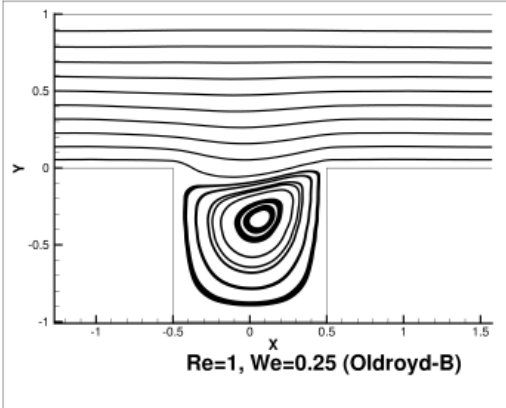

(c)

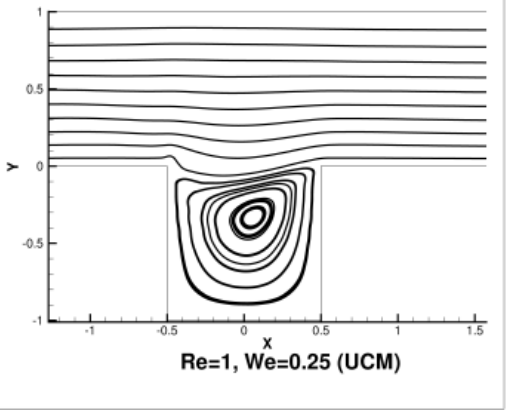

(f)

Fig. 3. Streamlines in the ALS- $S_{\text {von }}$ solutions at $R e=1$ for the Oldroyd-B model at (a) $W e=0.15$, (b) $W e=0.2$, and (c) $W e=0.25$ and the UCM model at (d) $W e=0.15$, (e) $W e=0.2$, and (f) $W e=0.25$.

We start with the uniform criss-crossed Mesh 1 with 8 partitions per unit length in Figure 1 (a). Based on the idea in [11], the mass conservation parameter $K=10^{6}$ is used in the LS formulations in the example. To illustrate the convergence of the LS method, streamlines of the method obtained using uniform Meshes 1 and 2 are plotted in Figure 1 (a) and (b); the figure shows convergent streamlines of the LS method for the Oldroyd-B model. Note that the refinement scheme for streamlines converges when $\left(\Delta \varepsilon_{n} / \Delta N_{n}\right)<10^{-4}$, where $\Delta \varepsilon_{n}$ and $\Delta N_{n}$ are the relative norm of the residual of the system in (2)-(6) and that of the element numbers of the mesh on every steps $n$, respectively. We demonstrate solutions obtained using the ALS-S $S_{\text {von }}$ and ALS methods by using two adaptive Meshes 3 and 4 generated using $\boldsymbol{\tau}_{v}$ and $|\mathbf{u}|_{2}$, respectively. Figure 1 indicates that two adaptive meshes are highly refined near the wall and reentrant corner (Figures 1 (c) and (d). Furthermore, the results reveal that streamlines on Mesh 3 and Mesh 1 are in agreement, and those

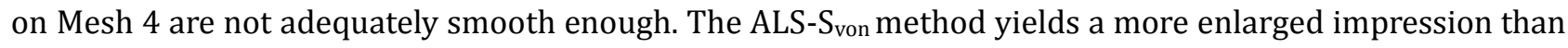
does the ALS method, which reveals that the refinement results are of high quality. Figure 2 shows profiles of $u(0, y)$ along the centerline obtained using the LS method on Meshes 1 and 2, the ALS-S $S_{\mathrm{vo}}$ method on Mesh 3, and the ALS method on Mesh 4. The profiles show that the results for Mesh 2 are closer to those for

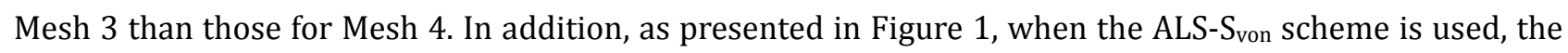
number of elements can be reduced from 24576 (Mesh 2) and 10421 (Mesh 4) to 9716 (Mesh3). These

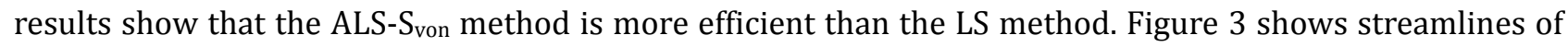
the ALS-S $\mathrm{S}_{\mathrm{von}}$ solutions for the Oldroyd-B and UCM models with $W e=0.15,0.2$, and 0.25 . Different vortex developing processes can be are observed in the figure. For low We fluids, we also observe that the vortex size increases as We increases, and the vortex moves toward the reentrant corners. These results are consistent with those obtained in [10]. Figure 4 (a) and (b) illustrates the effects of physical parameters on the horizontal velocity component, $u(0, y)$ along the vertical centerline with $W e=0.15,0.2$, and 0.25 for the Oldroyd-B and UCM models, respectively. The figure indicates that the fluid velocity near the top wall for 
$W e=0.25$ is higher than those for other $W e$ values, thus resulting in a flat velocity profile near the down wall.

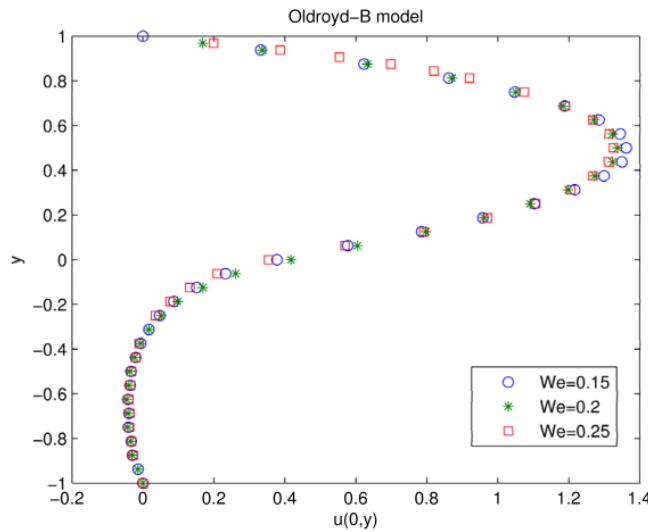

(a) Oldroyd-B model

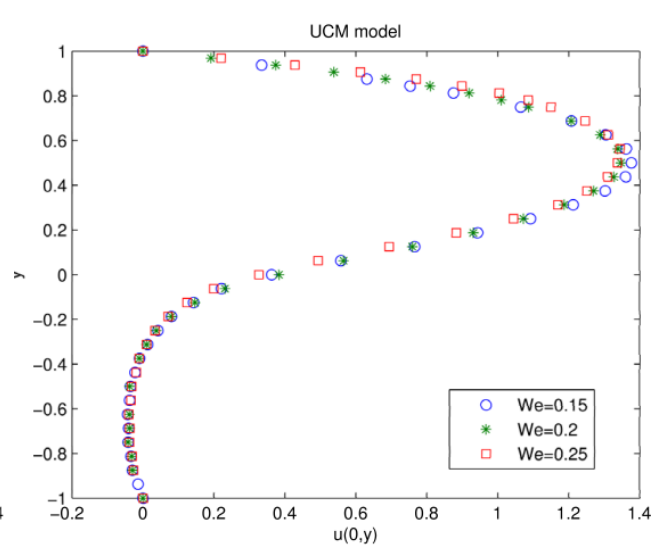

(b) UCM model

Fig. 4. Plots of $u(0, y)$ along the vertical centerline using the ALS-S $S_{\text {von }}$ solutions at $R e=1$ for the (a)

Oldroyd-B and (b) UCM models at $W e=0.15,0.2$, and 0.25 .

\section{Conclusion}

We present an adaptive mesh refinement strategy in the LS method for the Oldroyd-B and UCM viscoelastic models. The adaptive mesh approach is based on the von Mises stress. We show the adaptive LS method to the slot channel problem and address the physical parameter effects. Results obtained using the ALS-S $S_{\text {von }}$ method based on the von Mises stress outperform those obtained using a method based on the velocity magnitude, the refinement results of stress are able to capture streamline features for the effects of Weissenberg numbers, and satisfactory results are obtained using a lower total numbers of elements. In the work, we calculate an estimate of effective cost using the number of elements for different meshes. The processing time for different meshes may be considered and will be investigated in future.

\section{Acknowledgment}

This work was supported in part by the Ministry of Science and Technology of Taiwan under contract 104-2115-M-160-001-MY2.

\section{References}

[1] Lee, H. C. (2012). A nonlinear weighted least-squares finite element method for the Oldroyd-B viscoelastic flow. Appl. Math. Comput., 219, 421-434.

[2] Chen, T. F., et al. (2010). Least-squares finite element methods for generalized Newtonian and viscoelastic flows. Appl. Numer. Math., 60, 1024-1040.

[3] Bochev, P. B., \& Gunzburger, M. D. (1998). Finite element methods of least-squares type, SIAM, Revi., 40, 789-837.

[4] Chaudhry, J. H., et al. (2012). A weighted adaptive least-squares finite element method for the Poisson-Boltzmann equation. Applied Mathematics and Computation, 218, 4892-4902.

[5] Liu, J. L. (2000). Exact a posteriori error analysis of the least squares finite element method, Appl. Math. Comput., 116, 297-305.

[6] Cai, Z., \& Westphal, C. R. (2009). An adaptive mixed least-squares finite element method for viscoelastic fluids of Oldroyd type, J. Non-Newtonian Fluid Mech., 159, 72-80. 
[7] Lee, H. C. (2014). An adaptively refined least-squares finite element method for generalized Newtonian fluid flows using the Carreau model. SIAM J. Sci. Comput., 36, 193-218.

[8] Lee, H. C., \& Chen, T. F. (2015). Adaptive least-squares finite element approximations to Stokes equations. J. Comp. Appl. Math., 280, 396-424.

[9] Segalman, D., et al. (2000). Estimating the probability distribution of von Mises stress for structures undergoing random excitation. Journal of Vibration and Acoustics, 122, 42-48.

[10] Yin, H. J., et al. (2007). Numerical simulations of viscoelastic flows through one slot channel. Journal of Hydrodymanics, 19, 201-216.

[11] Lee, H. C. An adaptive least-squares finite element method for Giesekus viscoelastic planar contraction flows, submitted.

[12] Lee, H. C. (2015). A nonlinear weighted least-squares finite element method for the Carreau-Yasuda non-Newtonian model. J. Math. Anal. Appl., 432, 844-861.

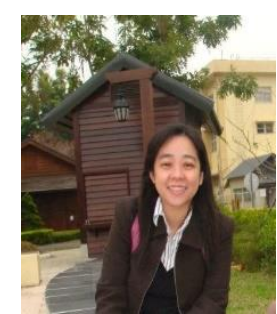

Hsueh-Chen Lee is the associate professor of General Education Center (GEC) at Wenzao Ursuline University of Languages in Taiwan. She was born in Kaohsiung on September 3rd of 1973. She received a Ph.D. degree of Mathematics from National Chung Cheng University in 2008.

She is a chairperson of GEC since 2016, and also served as a manager of Academic Development Section on Research and Development from 2012 to 2013. Her research interests are numerical analysis such as numerical PDE for flow problems and adaptive mesh development. Prof. Lee is a member of the Mathematical Society of Republic of China (TMA), Taiwan SIAM (TWSIAM). She has received many awards including the financial support from the Ministry of Science and Technology of Taiwan form 2014 to 2017. 